EPJ Web of Conferences 19, 01006 (2012)

DOI: $10.1051 /$ epjconf/20121901006

(C) Owned by the authors, published by EDP Sciences, 2012

\title{
Rotation curve of the Milky Way
}

\author{
O. Golubov ${ }^{1, a}$, A. Just ${ }^{1}$, T.C. Beers ${ }^{2}$ and Y.S. Lee ${ }^{2}$ \\ ${ }^{1}$ Astronomisches Rechen-Institut, Zentrum für Astronomie der Universität Heidelberg, \\ Mönchhofstraße 12-14, 69120 Heidelberg, Germany \\ ${ }^{2}$ Department of Physics \& Astronomy and JINA (Joint Institute for Nuclear Astrophysics), \\ Michigan State University, East Lansing, MI 48824, USA
}

\begin{abstract}
We use SEGUE and Hipparcos data to restrict the behaviour of the rotation curve of the Milky Way in the solar neighbourhood. Then we construct a density model of the Milky Way which best reproduces the available observations of the rotation curve and is consistent with the density constraints in the solar neighbourhood.
\end{abstract}

\section{INTRODUCTION}

The rotation curve gives a powerful tool to study the mass distribution in the Milky Way. An extensive compilation of observational data is given in [6]. But the density model proposed in [6] is inconsistent with local density and dark matter density constraints in the solar neighbourhood [4]. We reanalyse these observational data, supplement them with local constraints and with our own analysis of the behaviuor of the rotation curve in the solar neighbourhood, and construct a density model of the Galaxy.

\section{THE BEHAVIOUR OF THE ROTATION CURVE IN THE SOLAR NEIGHBOURHOOD}

In our analysis we use two samples of stars: SEGUE G-dwarfs, whose distances were determined in [5], and GCS stars with distances from Hipparcos [2].

For each sample we calculate the mean rotational velocity $\bar{v}_{\phi}$ and correct it for the asymmetric drift with the aid of the equation [1]

$$
v_{c}^{2}=\bar{v}_{\phi}^{2}+\sigma_{\phi}^{2}+2\left(\frac{R}{R_{d}}-1\right) \sigma_{R}^{2}+\sigma_{z}^{2}
$$

This equation assumes constancy of the shape of the velocity ellipsoid, its alignment with the axes of spherical coordinate system, and an exponential disc with scalelength $R_{d}$ and a constant thickness.

Thus we find the circular velocity $v_{c}$ for our samples. We assume the local circular velocity $v_{c \odot}=231 \mathrm{~km} / \mathrm{s}$, the local standard of rest from [3], and the disc scalelength $R_{d}=2.5 \mathrm{kpc}$. The resulting rotation curve is presented in the left panel of Fig. 1. SEGUE sample is plotted with purple line, GCS is separated in 5 colour bins, which are plotted with points of different colours. Mean rotational velocities without correction for asymmetric drift being implemented are plotted with dashed lines, the corrected circular velocity with solid lines. The consistency of the circular velocity for GCS colour bins with different mean velocities demonstrates viability of the adopted value of $R_{d}$ and other our assumptions. The rotation curve for the SEGUE data appears to be essentially flat in the solar neighbourhood. At least, it obviously demonstrates no drastic dip between 8 and $10 \mathrm{kpc}$, as it was assumed in [6].

\footnotetext{
ae-mail: golubov@ari.uni-heidelberg.de
}

This is an Open Access article distributed under the terms of the Creative Commons Attribution-Noncommercial License 3.0, which permits unrestricted use, distribution, and reproduction in any noncommercial medium, provided the original work is properly cited. 

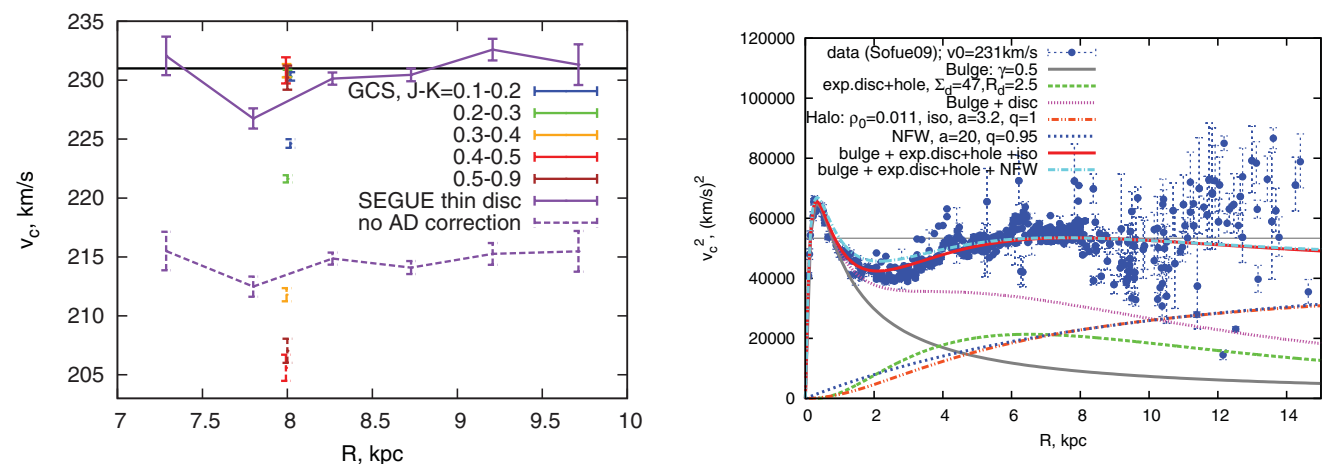

Figure 1. The left panel shows the rotation curve for SEGUE and GCS stellar samples. The right panel shows $v_{c}^{2}$ for our theoretical models overplotted with the observational data from [6].

Measuring the ratio of velocity dispersions in the direction of the galactic rotation and in the radial direction can serve as another probe of the rotation curve. If we assume the rotation curve to be given by a power law $v_{c} \propto R^{\alpha}$, then the epicyclic theory [1] leads to the following expression,

$$
\frac{\overline{\left(v_{\phi}-v_{c}\right)^{2}}}{\sigma_{R}^{2}}=\frac{R}{2 \Omega} \frac{\partial \Omega}{\partial R}+1=\frac{\alpha+1}{2} .
$$

We measure this ratio of velocity dispersions, getting $\alpha=-0.04$ for SEGUE and $\alpha=-0.02$ for GCS. This also implies that the rotation curve in the solar neighbourhood is nearly flat.

\section{DENSITY MODEL OF THE MILKY WAY}

We construct a 3-component density model of the Milky Way as a sum of Dehnen bulge, an exponential disc with a hole, and a flattened dark matter halo with either cored isothermal or NFW profile. We adjust the free parameters in such a way, to get the best fit of the observational data [6] and a flat rotation curve in the solar neighbourhood. When adjusting the parameters we constrain ourselves with the local surface density of the disc $\Sigma_{\text {disc }}=45.2 \pm 4 \mathrm{M}_{\odot} / p c^{2}$ and the local volume density of the dark matter halo $\rho_{h, 0}=0.014 \mathrm{M}_{\odot} / \mathrm{pc}^{3}[4]$.

Thus we get the density model whose rotation curve is presented in the right panel of Fig. 1. The density of the bulge is given by Dehnen model with power index $\gamma=0.5$, total mass $M_{b}=$ $1.8 \times 10^{10} \mathrm{M}_{\odot}$, and the scale radius of $a_{b}=0.22 \mathrm{kpc}$., The disc is exponential with $R_{d}=2.5 \mathrm{kpc}$ with a hole of a twice smaller scalelength with $\epsilon=0.4$. Two different models of dark matter halo are considered, spherical cored isothermal halo with core radius $a=3.2 \mathrm{kpc}$, and flattened NFW profile with $a=20 \mathrm{kpc}$. Both models succeed to reproduce the data, with a somewhat better fitness for the one with cored isothermal profile.

\section{References}

[1] Binney, J., Tremaine, S., Galactic Dynamics (Princeton University Press, Princeton 2008), pp. 170, 355

[2] Holmberg, J., Nordström, B., Andersen, J., A\&A 501, 941 (2009)

[3] Dehnen, W., Binney, J., MNRAS 398, 387 (1998)

[4] Just, A., Jahreiß, H., MNRAS 402, 461 (2010)

[5] Lee, Y.S., Beers, T.C., An, D., Ivesic, Z., Just, A. et al. ApJ 738, 187 (2011)

[6] Sofue, Y., Honma, M., Omodaka, T., PASJ 61, 227 (2009) 